Proceedings of the 2011 Winter Simulation Conference

S. Jain, R.R. Creasey, J. Himmelspach, K.P. White, and M. Fu, eds.

\title{
SIMULATION AND ANALYSIS OF RAILROAD HUMP YARDS IN NORTH AMERICA
}

\author{
Edward Lin \\ Clark Cheng \\ Norfolk Southern Corporation \\ 1200 Peachtree St. NE \\ MS 12-117 \\ Atlanta, GA 30309, USA
}

\begin{abstract}
Freight railroad terminals receive inbound trains, classify or regroup railcars, and build outbound trains. There are two types of terminals: hump yard which uses gravity to sort railcars and flat switching yard. In general, a hump yard is more productive than a flat switching yards. Due to the complexity of terminal operations, computer simulation offers a flexible and credible technique to identify opportunities for yard performance improvements. However, the use of simulation technique to model terminal operations is not a common practice in freight railroads. In this paper, we introduce a simulation model which depicts the typical operations in a railroad hump yard and present key performance measurements that are used to gauge the efficiency of yard operations and infrastructure. Finally, we illustrate the use of simulation model to improve terminal operations.
\end{abstract}

\section{INTRODUCTION}

Line and terminal simulation models provide a means to analyze the capacity and/or operating performance of the rail network under a variety of condition (Krueger et al. 2000). Dalal and Jensen (2001) stated that one of the major barriers to the widespread use of simulation in railroads is a general lack of awareness of the technology. Rail Traffic Controller (RTC) from Berkeley Simulation Software is an industry-standard simulation software for mainline simulation. However, terminal simulation appears to be far lagging behind in railroads. Lin and Cheng (2009) described a yard simulation framework and its implementation in a North American railroad.

For a given terminal physical layout and operating business rules and policies, terminal simulations focus on studying impact of inbound and outbound traffic with respect to the terminal capacity. In this type of study, terminal layout is modeled. Other inputs include a train plan with arrival and departure train schedules, terminal resources, and traffic mix. Terminal simulation models can assist in identifying bottleneck, car delays and process improvement. This type of simulation study is extremely useful in designing train plan to assess if the terminal has capability to handle traffic. In addition, by changing terminal physical layout (e.g. adding additional tracks), terminal simulation model can also be used in assessing major capital investment and new handling methodologies in the planning stage.

The objective of this paper is to introduce a terminal simulation model and presents key performance measures that can be used for terminal operating and/or infrastructure improvement. First, we give an overview of typical terminal operations in a rail hump yard. Then, we present a terminal simulation model and its input data model and key performance metrics. Finally, we illustrate the use of some statistics for bottleneck analysis. 


\section{Lin and Cheng}

\section{BACKGROUND}

In railroad freight transportation, a shipment to be moved from its origin to destination is carried by a rail car that meets its characteristics. When demand is high, a set of shipments with the same origins and destinations are grouped together in blocks and moved directly from origin to destination. Railroad runs trains, namely unit trains, to carry those cars. However, when demand is not sufficient, shipments are consolidated and carried through a sequence of trains. Typically, cars with different final destinations but sharing a portion of their trips are assembled into blocks. Cars in the same block travel together in railroad network in order to increase efficiency and reduce intermediate handlings. At an intermediate handling location, cars are re-sorted (or classified) for their next destination in the trip. A railroad facility that receives trains, classifying cars, assembly and outbound trains is called a terminal. Classification yard (or bowl) of a terminal consists of a set of classification tracks that are used to sort or classify cars. Wang et al. (1981) described a methodology to design a rail yard.

Figure 1 shows a hump yard that contains a receiving yard where trains are received, a classification yard (also known as a bowl), a forwarding yard where outbound trains are assembled and depart, and pull back tracks that are used to move cars from classification yard to forwarding yard.

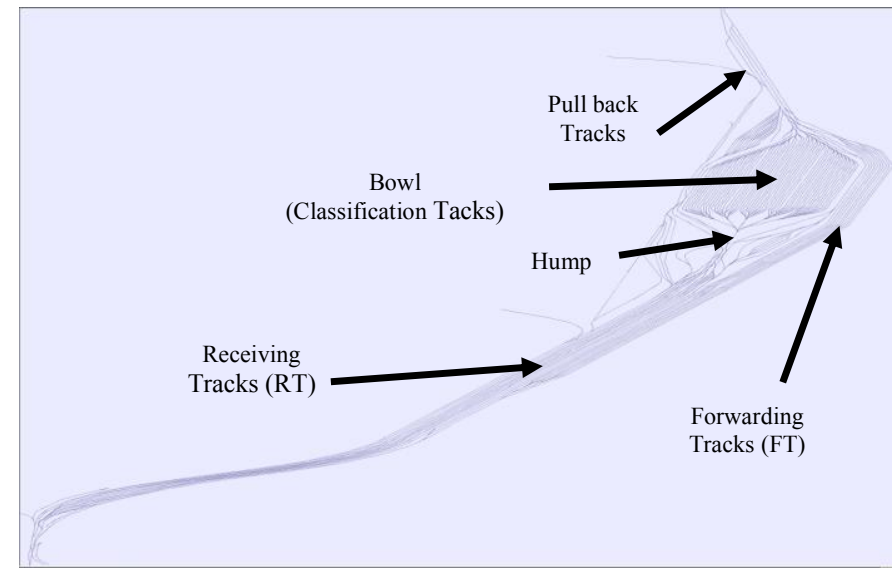

Figure 1: A hump yard (Lin and Cheng 2009)

When an inbound train arrives at a hump yard, the terminal trainmaster instructs the train crew to park cars in the receiving yard. After a train comes to a completely stop at a receiving track, the engineer makes a reduction on all cars. The conductor or utility man ties the hand brakes down on 5 cars. The locomotives are un-coupled and move to fueling platform. The cars may go through inbound car inspection process at the receiving yard by mechanical crew, and then the air on each car is bled out to release the air brake by utility man. When all cars on a receiving track is ready for humping, yard master dispatches a locomotive engine (also known as a hump engine) to couple cars. Next step is humping, a process in which a hump engine pushes cars from a track in the receiving yard over the hump. When cars arrive at the hump, the top of the hill, pins are pulled and cars roll down by the force of gravity to assigned classification tracks in the bowl. Retarders control car movement speeds to make sure that they have enough velocity to reach their assigned tracks, but not damage the already parked cars. Depending on shipment characteristics and destinations, several cars may be roll together as a group. Ideally, each classification track is designated to an outbound block. But due to the limited number of classification tracks, several outbound blocks can be assigned to one classification track. Those cars may need to be re-classified (or re-hump) later on when tracks become available. Cars are waiting on the classification tracks for departure trains. When it is time to assemble an outbound train, outbound blocks are pulled from classification tracks and assembled into the outbound train. The list of potential blocks that each outbound train can carry is specified in the operating plan. The sequence of blocks on an outbound train is called block standing 


\section{Lin and Cheng}

order. The blocking standing order determines the sequence of cars that need to be pulled from classification tracks. A pull back engine first pulls the cars for the first block onto a pullback lead track, and then shovels them to the clear point of a departure track in the forwarding yard, unties two hand brakes, couples with the cut, pushes all cars beyond the clear point, ties two hand brakes, uncouple pullback engine, and move the pullback engine out of the forwarding track to next job. This process is repeated until all cars for the outbound train are assembled. After a train is assembled, the cars must be inspected for mechanical failure and defects and a brake test must be performed before the train can leave the yard. If a car is identified as a bad order, it must be set out from the train and sent to a bad-order track for repair.

Figure 2 shows a typical process flow in a hump yard. Rectangular boxes represent processes and triangular shapes represent receiving/classification/forwarding yards or buffers. Dirnberger and Barkan (2006) noted from literatures that majority of time cars spent in terminal (about $77.3 \%$ ) is non-valueadded idle time, waiting for next process.

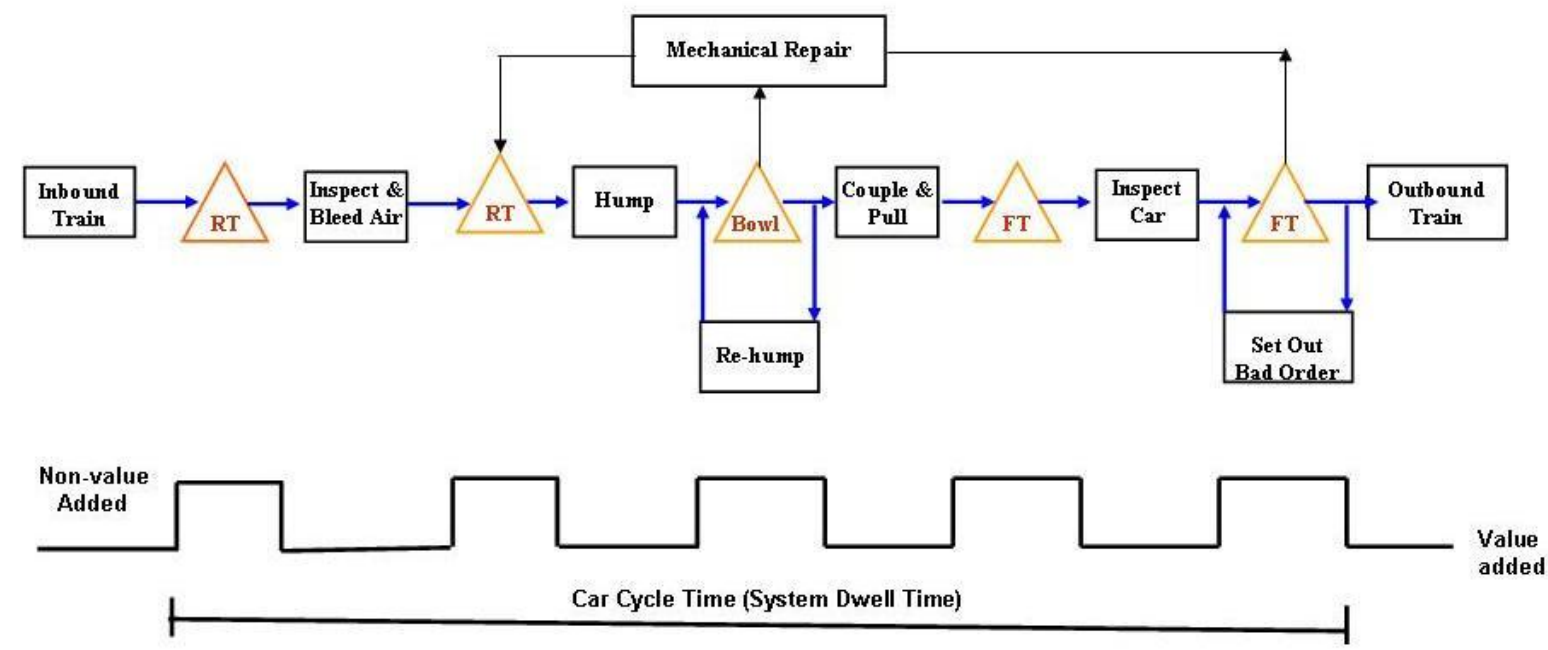

Figure 2: Typical process flow of hump yard

\section{THE HUMP YARD SIMULATION MODEL}

Due to the complexity of yard operations and management, a rail yard operations planning and management tool to support yard capacity and improvement studies is needed (Lin and Cheng 2009). A yard capacity study is to assess how many cars, blocks and trains can be handled with the existing infrastructure and resources. Rail yard improvement studies may include infrastructure improvement (e.g., adding a new track or crossover), resource requirements (e.g., number of yard engine and crews), and capability to handle increased traffic and trains.

Figure 3 shows typical inputs and outputs of a rail yard simulation model. In the following sections, key inputs and outputs are described in details. 


\section{Lin and Cheng}

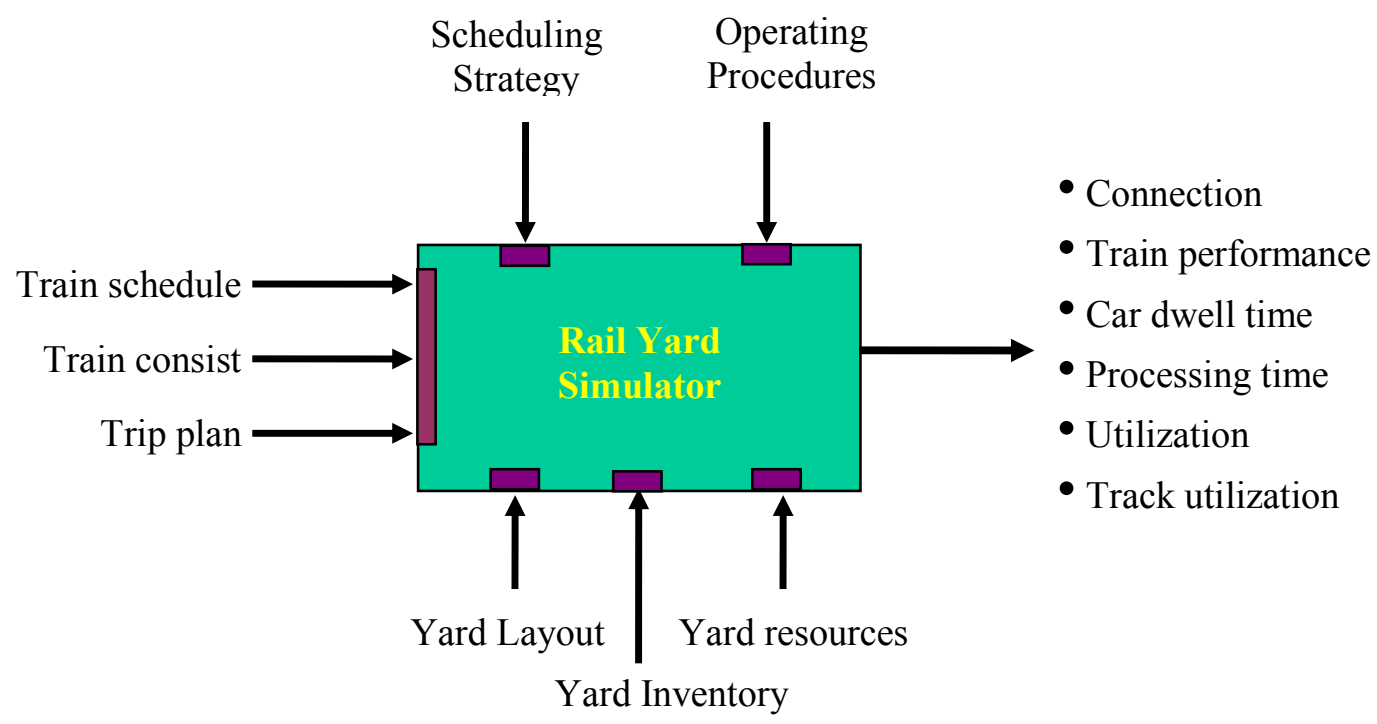

Figure 3: Rail yard simulation model

\subsection{Yard Layout}

CAD drawings are commonly used to represent yard layout. The CAD data can be interpreted as nodes and lines. The lines can be straight lines or poly lines. A rail yard layout is represented as $\mathrm{G}=(\mathrm{V}, \mathrm{A}, \mathrm{S}, \mathrm{K}$, $\mathrm{P}, \mathrm{W} 3, \mathrm{~W} 4)$, where $\mathrm{V}$ is a set of nodes (or points), $\mathrm{A}$ is a set of lines (straight lines or poly lines), $\mathrm{S}$ is a set of switches $(\mathrm{S} \subset \mathrm{V}), \mathrm{K}$ is a set of functional tracks, $\mathrm{P}$ is a finite set of ordered lines, W3: $(\mathrm{K} * \mathrm{~K}) \rightarrow \mathrm{N}$ defines feasible immediate movement between a pair of tracks, and $\mathrm{W} 4:(\mathrm{K} * \mathrm{P}) \rightarrow \mathrm{N}$ defines the physical tracks of a functional track. Common functional tracks in a hump yard includes receiving tracks, classification tracks, forwarding tracks, local yard, hump, hump leads, pullback leads, and mainline. Switches include manual switches and power switches.

\subsection{Train Schedule}

Terminal Clock is generally used to denote train schedule in a terminal, which specifies timing of inbound and outbound trains. Additional train attributes are also required for terminal simulation, e.g. train direction, train capacity, train block standing order.

\subsection{Trip Plan}

U.S. North American freight railroads moved from tonnage-based dispatching to schedule-based dispatching. In tonnage-based dispatching, trains are only run when enough traffic are accumulated. In scheduled-based dispatching, there is a plan for every car. The plan (or trip plan) specifies the terminals a car is going to or going through and every train the car is going to ride on from its origin to destination. Specifically, from a terminal view, a trip plan of a car specified the arrival time of the inbounding train and departure time of departure train of the car. Trip plan is the base for a key terminal performance measure, car connection measurement, see 1 for definition of measurement.

\subsection{Train Consist}

Based on trip plan and train blocking standing order, train consist shows a list of all the cars in a train in standing order from locomotive to End-of-Train-Device (EOTD). 


\section{Lin and Cheng}

\subsection{Typical Performance Measures}

1. Connection $(\%)$

Connection performance measures the percentage of connections made as scheduled (right train and right date), advanced (right train and earlier date), or missed (right train and later date or wrong trains).

2. Outbound Train On-time Performance (\%)

Train performance measures the percentage of scheduled outbound trains departing on-time at a terminal.

3. Resource Utilization (\%)

Typical resources in a terminal include hump engines, pullback engines, utility man, and mechanical crew. Resource utilization measures percentage of times resources spent in each state. For example, a hump engine states include light engine move, busy, waiting, and idle.

4. Hump Count and Occupancy (\%)

Hump count is a classical measurement in a hump controller, which measures number of cars have been humped in a time frame. Typical statistics are collected at each shift. Hump occupancy or hump utilization measures the percentage of times that hump is in busy state.

5. Humping and Pullback Process Cycle Time

Humping or pullback process cycle time measures average processing time of hump work orders or pullback work orders.

6. Terminal Dwell Time (hrs)

Terminal dwell, expressed in hours, is the average time a car resides at a terminal. The measurement begins with a train inbounded event and ends with a train departure event. In other words, terminal dwell time is calculated as the time difference of a car between its inbounded time and departure time.

In order to analyze car movements in details, terminal dwell time is further divided into three stages: dwell time at receiving yard, dwell time at classification yard, and dwell time at forwarding yard. Dwell time of a car at receiving yard is calculated as the time difference between its inbounded time and its start moving time from RT. Dwell time of a car at classification yard is the time difference between the time it reaches a classification track and its pull time. Dwell time of a car at forwarding yard is the time difference between the time it is pushed to a forwarding track and its departure time.

Average dwell time is the summation of dwell time of each car divided by total number of cars. Terminal dwell time is the summation of dwell time at each yard and processing times. The processing times include humping process time and pullback process time.

7. Track Utilization (\%)

Track utilization measures the track capacity utilization. In general, it can be expressed in three perspectives: track length, number of used tracks, and number of clear tracks.

\section{BOTTLENECK ANALYSIS AND DISCUSSION}

Dirnberger and Barkan (2006) indicated that pullback process is the bottleneck in yard operations based on various published studies. The pullback process consists of blocks of cars being pulled from the classification tracks (bowl) and placed together to form outbound trains in the forwarding yard. A pullback process starts with moving a pullback engine from either an idle position at yard or a forwarding track in 


\section{Lin and Cheng}

the forwarding yard to classification yard (bowl), coupling with cars on a classification track or coupling cars on several classification tracks if it is a multiple pulls, moving pulled cars to a pullback lead, waiting at the pullback lead, and moving pulled cars to a forwarding track to form a train.

Figure 4 shows an example of average percentage of times spent in each state of pullback processes. Waiting time states are non-value added state. Those states need to be examined closely to improve pullback performance.

Figure 5 shows typical pullback performance measures: number of pull cars and number of pullback processes. These two measures might be good indicators for model validation.

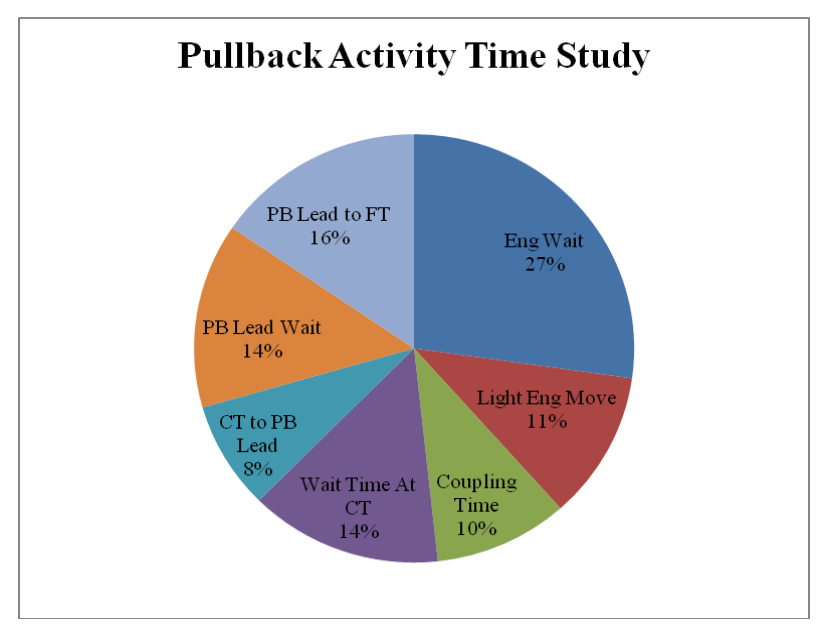

Figure 4: Pullback activity time analysis

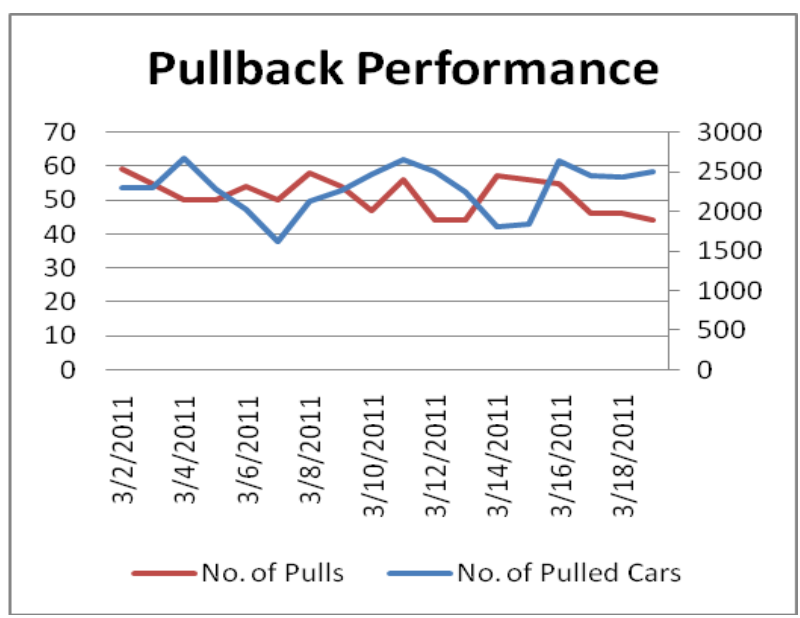

Figure 5: Pullback Activity Analysis

Though the pullback process is the bottleneck, the humping process should be managed to make pullback process as efficient as possible. In practice, hump performances are merely measured on number of humped cars and hump occupancy. However, poor humping strategy can easily lead to poor pullback performance. Ideally, a good humping strategy should properly sort cars in the bowl, which leads to a better bowl quality. In the following, we illustrate complexity of humping strategy by showing the relationships between bowl quality and humped cars and blocks. First, we present defintions to measure bowl quallity.

Definition 1 A clear track is a classification track that has no cars on it. 


\section{Lin and Cheng}

Definition 2 A clean track is a classification track that has only one block or class code or destination.

Definition 3 A layer track is a classification track that has more than one block or class code, however, the cars are positioned in proper order following their train departure time. If blocks of cars will depart on the same outbound train, then blocks follow blocking standing order of the outbound train.

Definition $4 \mathrm{~A}$ dirty track is a classification track that blocks of cars are not in proper order.

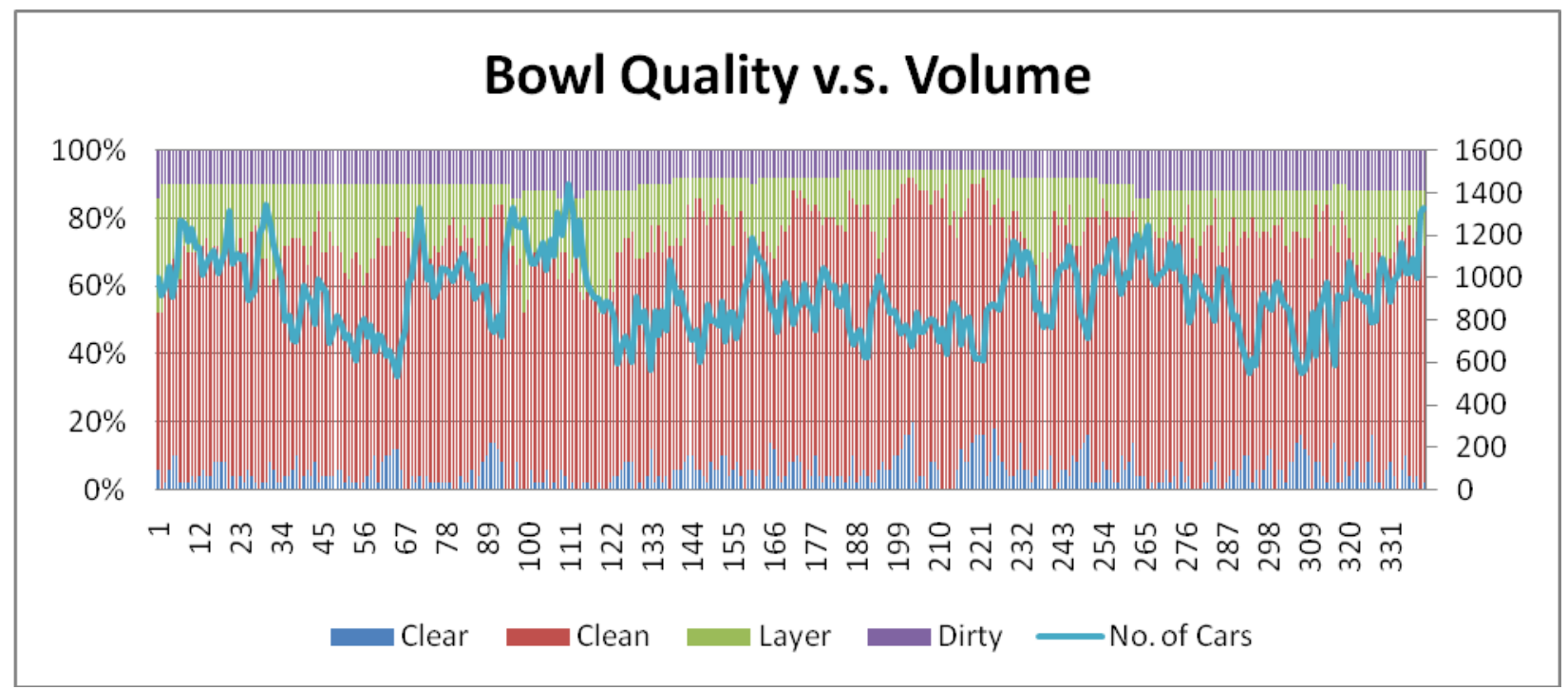

Figure 6: Bowl Quality Analysis vs. Bowl Volume

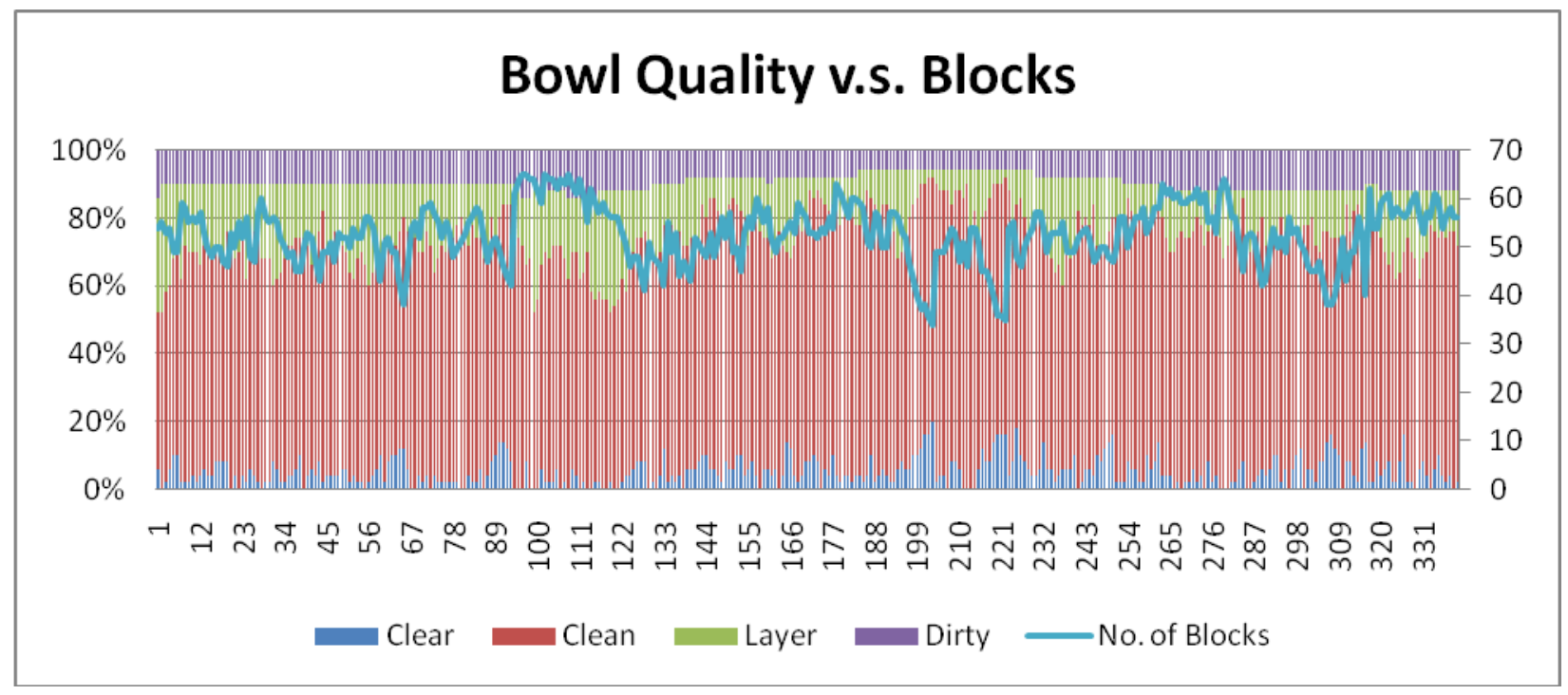

Figure 7: Bowl Quality Analysis vs. Blocks

Dirnberger and Barkan (2006) proposed a method to measure the quality of sort metric. As a matter of fact, the formula measures Incorrect Sort Rating (ISR) and is built in three levels: car, track and bowl. However, due to traffic mix, hump yards typically handle more blocks codes than number of available classification tracks. Sort cars to dedicate track or group based on static track allocation or static group schema may not always be feasible. Daganzo (1987) described dynamic blocking strategies that require fewer classification tracks, but more switches than their static counterparts. 


\section{Lin and Cheng}

Figure 6 shows the relationship between bowl quality and bowl volume. $\mathrm{X}$-axis of Figure 6 is the time instances that the bowl snapshots are taken. Left y-axis is the \% of classification tracks in each track category. Right $y$-axis is the total number of cars in the bowl. Figure 7 shows the relationship between bowl quality and number of blocks in the bowl. X-axis of Figure 7 is the time instances that the bowl snapshots are taken. Left y-axis of Figure 7 is the \% of classification tracks in each track category. Right $y$-axis of Figure 7 is number of blocks in the bowl. In observations, high bowl volume and high number of blocks lead to higher number of dirty tracks.

\section{CONCLUSION}

This paper introduces a rail yard simulation model and presents an input data model of the rail yard simulation and key performance measures. The simulation model can be used for terminal operating and infrastructure improvement studies. An example of using some statistics to analyze bottleneck in a yard is illustrated. Future research will focus on developing methodology to identify terminal capacity.

\section{ACKNOWLEDGMENTS}

The authors would like to acknowledge the contributions of the project team members: Rajesh Kalra, Dr. Vinay Mehendiratta, Pratik Singh, and Johnny Wang.

\section{REFERENCES}

Berkeley Simulation Software, LLC. 2011. "Rail Traffic Controller." Accessed June 14, 2011. http://berkeleysimulation.com.

Daganzo, C. F. 1987. "Dynamic Blocking for Railyards: Part I. Homogeneous Traffic." Transportation Research 21B:1-27.

Dalal, M. A. and L. P. Jensen. 2001. "Simulation Modeling at Union Pacific Railroad." In Proceedings of the 2001 Winter Simulation Conference, edited by B.A. Peters, J.S. Simth, D.J. Medeiros, and M. W. Rohrer, 1048-1055. Piscataway, New Jersey: Institute of Electrical and Electronics Engineers, Inc.

Dirnberger, J. R. and C. P. L. Barkan. 2006. "Implementing Bottleneck Management Techniques and Establishing Quality of Sort Relationships to Improve Terminal Processing Capacity." In Proceedings of the $7^{\text {th }}$ World Congress on Railway Research, Montreal.

Krueger, H., E. Vaillancourt, A. M. Drummie, S. J. Vucko and J. Bekavac. 2000. "Panel Discussion: Simulation Within the Railroad Environment." In Proceedings of the 2000 Winter Simulation Conference, Edited by J. A. Joines, R. R. Barton, K. Kang, and P. A. Fishwick, 1191-1200. Piscataway, New Jersey: Institute of Electrical and Electronics Engineers, Inc.

Lin, E. and C. Cheng. 2009. "YardSim: A Rail Yard Simulation Framework and its Implementation in a Major Railroad in the U.S." In Proceedings of the 2009 Winter Simulation Conference, Edited by M. D. Rossetti, R. R. Hill, B. Johansson, A. Dunkin and R. G. Ingalls, 2532-2541. Piscataway, New Jersey: Institute of Electrical and Electronics Engineers, Inc.

Wang, P. J., M. Sakasita, W. A. Stock, C. V. Elliott, and M. A. Hackworth. 1981. "Railroad Classification Yard Technology Manual - Volume I: Yard Design Methods.” Technical Report No. 6364, SRI International, Menlo Park, California.

\section{AUTHOR BIOGRAPHIES}

EDWARD LIN manages operations research projects at Norfolk Southern Corporation. He received his $\mathrm{Ph} . \mathrm{D}$. in industrial and systems engineering from the Georgia Institute of Technology. He has 17 years of research and development experience in academic, software, and transportation. His research interests include operations research, simulation, rule-based applications in transportation and manufacturing industries. His email address is <edward.lin@nscorp.com>. 


\section{Lin and Cheng}

CLARK CHENG is the director of the Operations Research group at Norfolk Southern Corporation, a Class I railroad in the U.S. He has been working in the railroad industry for 17 years and has participated in developing and implementing computer models and decision support systems for locomotive dispatching, car scheduling, network optimization, fleet planning, demand forecasting, yard simulation, crew assignments, service design, and car distribution. He received his Ph.D. degree in industrial engineering from Clemson University. His email address is<clark.cheng@nscorp.com>. 